\title{
The vocal tract and the sound of a didgeridoo
}

\section{Acoustic measurements show how a player can extract a range of timbres from this primitive instrument.}

The Australian didgeridoo (or yidaki in the Yolngu language of northern Australia) is a simple musical instrument that, at the lips of an experienced player, is capable of a spectacular variety of timbres - considerably greater than those that can be coaxed from orchestral instruments, for example. To understand this phenomenon, we simultaneously measured the sound produced by the didgeridoo and the acoustic impedance of the player's vocal tract. We find that the maxima in the envelope of the sound spectrum are associated with minima in the impedance of the vocal tract, as measured just inside the lips. This acoustic effect is similar to the production of vowel sounds made during human speech or singing', although the mechanism is different, and leads to the surprising conclusion that experienced players are subconsciously using their glottis to accentuate the instrument's tonal variation.

The didgeridoo is traditionally made from a small tree trunk whose core has been eaten by termites. It usually plays only one note, with the player's lips vibrating at a frequency near a maximum in the instrument's acoustic impedance $^{2}$, which is a measure of how difficult it is to produce air vibration at a given frequency. Its musical value comes from the great variety of timbres and rhythms produced by experienced players as they alter their tongue position and mouth geometry.

Characteristic bands of emphasized frequencies, or formants, occur in the output sound and are similar to the higher formants of spoken vowels ${ }^{3}$. The acoustic mechanism responsible for the strong formant production has hitherto not been well understood because of the complexity of the interactions between the vocal tract, the lips and the instrument. The acoustic properties of the tract are particularly difficult to measure during performance because of the very high sound levels inside the mouth.

In these experiments, a broadband acoustical current was injected into the player's mouth, just inside the lips, using a tube (diameter, $3.7 \mathrm{~mm}$ ). A probe microphone next to it (diameter, $1.5 \mathrm{~mm}$ ) measured the pressure. The acoustic impedance was calculated as described ${ }^{45}$.

Figure 1 shows an example of the vocal-tract impedance. The spectrum of the radiated sound was measured immediately after the impedance measurement, with the player maintaining a constant sound but with no injected broadband current, to avoid this

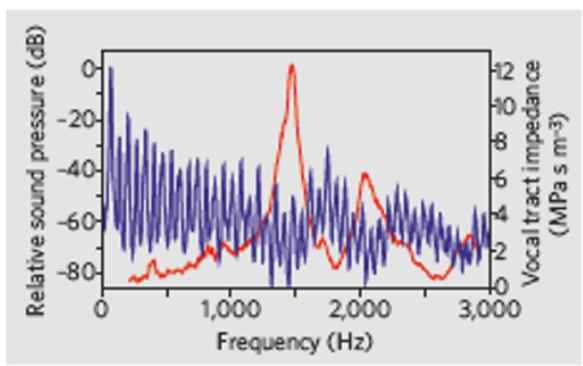

Figure 1|Acousticmeasurements froma didgeridooperformance. Spectrum of radiated sound (blue) and the magnitude of acoustic impedance of the vocal tract (red) measured just inside the lips of a didgeridoo player during performance. The player performs the 'high drone', produced with the tongue close to the hard palate, which generates a characteristic strong formant at $1.8 \mathrm{kHz}$. Similar measurements with the tongue in the low position revealed no strong impedance maxima and no strong formants. (For sound file and spectra ${ }^{8}$, see supplementary information.)

appearing as noise background in the sound spectrum. The sound output is reduced for frequencies at which the vocal-tract impedance is sufficiently large, and the acoustic flow through the lips is inhibited. For frequencies at which the vocal-tract impedance is small, flow into the instrument is greater and more sound is produced. Results were similar on other didgeridoos and with other players. (For details, see supplementary information.) A linear regression of the frequencies of formant maxima/minima against impedance minima/ maxima has a slope of 0.93 , with correlation coefficient of 0.98 (46 measurements from three players, results not shown).

These results indicate that strong formants in the sound depend on the presence of strong resonances in the tract, and this in general requires the glottis to be partially closed to enhance reflection and to prevent the resonant highfrequency components being absorbed in the resistive impedance of the lungs. We conclude that a major difference between a novice and an experienced player is a learned, but usually subconscious, ability to reduce the glottal opening (a similar difference has been proposed for brass-instrument players $\left.{ }^{6}\right)$. The effect of tongue position on the pitch and timbre of orchestral lip-valve instruments is much more modest because of their relatively narrow bore, but is still musically significant'. An understanding of tract-lip-bore interactions in the didgeridoo, where the effects are pronounced, is likely to lead to an improved understanding of these more subtle effects in orchestral instruments.

Alex Tarnopolsky ${ }^{\star}$, Neville Fletcher ${ }^{\star} \dagger$,

Lloyd Hollenbergt, Benjamin Lange*, John Smith, Joe Wolfe ${ }^{\star}$

*School of Physics, University of New South Wales, Sydney, New South Wales 2052, Australia e-mait j.wolfe@unsw.edu.au

†Research School of PhysicalSciences, Australian National University, Canberra 0200, Australia $\ddagger$ School of Physics, University of Melboume, Parkville, Victoria 3010, Australia

1. Sundberg 1 The Sclence of the Singing Voice (Northem Iilinois UniN $N_{7}$ De Kall, 1987).

2. Fletcher, N.H.\& Rossing, T.D. The Physics of Musical instruments 2 nd edn (Springec, New York 1998).

3. Fletcher, N. H. Austr. Aboniginal Stud 1, 28-37 (1983).

4. Epps, L, Smith, 1 R. \& Wolfe, 1 Meas Sci. Technol. 8, III2-1121(1997).

5. Smith I. R, Henrich, N \& Wolfe, I.Proc. Inst. Acoust 19, 315-320 (1997).

6. Mukai, S L L Otolaryngal. Jpn 92,260-270 (1989).

7. Wolfe, 1 , Tarnopolsky A.Z, Fletcher, N.H, Hollenberg, LC. L.\&Smith, L Proceedings of the Stockhalm Music Acoustics Conference (ed Bresin R) 307-310 (2003). 8. Music A coustics wwwphysunsweduau/ - w/ widakihtml

Sup plementary information accompanies this commun ication on Nature's webs ite. Competing fin ancial interests: declared none. doi:10.1038/43639a

\section{PALAEOCLIMATOLOGY}

\section{The record for marine isotopic stage 11}

The marine isotopic stage 11 (MIS 11) is an extraordinarily long interglacial period in the Earth's history that occurred some 400,000 years ago and lasted for about 30,000 years. During this period there were weak, astronomically induced changes in the distribution of solar energy reaching the Earth. The conditions of this orbital climate forcing are similar to those of today's interglacial period ${ }^{12}$, and they rendered the climate susceptible to other forcing - for example, to changes in the level of atmospheric carbon dioxide. Here we use 